\title{
Accrued HIV evidence turns treatment dogma on its head
}

\section{Erika Check, Washington}

A series of studies has dispelled the widespread notion that patients who don't take every dose of their anti-HIV medication create a public-health risk by helping to nurture HIV strains that resist therapy.

The findings suggest instead that some patients who do not take all of their medicine are actually less likely to become resistant to therapy than those who adhere rigidly to their doctors' instructions.

This fresh consensus has implications for AIDS-treatment plans in poor countries, where drug companies, in particular, have often argued that HIV patients are less likely to adhere to such instructions than those in rich countries. Such 'low adherence' had been thought to encourage the success of mutations that would make the patients' viruses more resistant to treatment.

"The relationship between adherence and resistance is more complex than we previously understood," says David Bangsberg, a clinician at the University of California, San Francisco, who published a study this week showing that HIV-positive patients who were having trouble subduing the virus were more likely to develop resistance to their medications if they took most of their prescribed doses (D. R. Bangsberg et al. AIDS doi:10.1097/01.aids. 0000076320.42412.fd;2003).

Bangsberg warns, however, that his and others' findings do not mean that patients should deliberately skip doses, because the

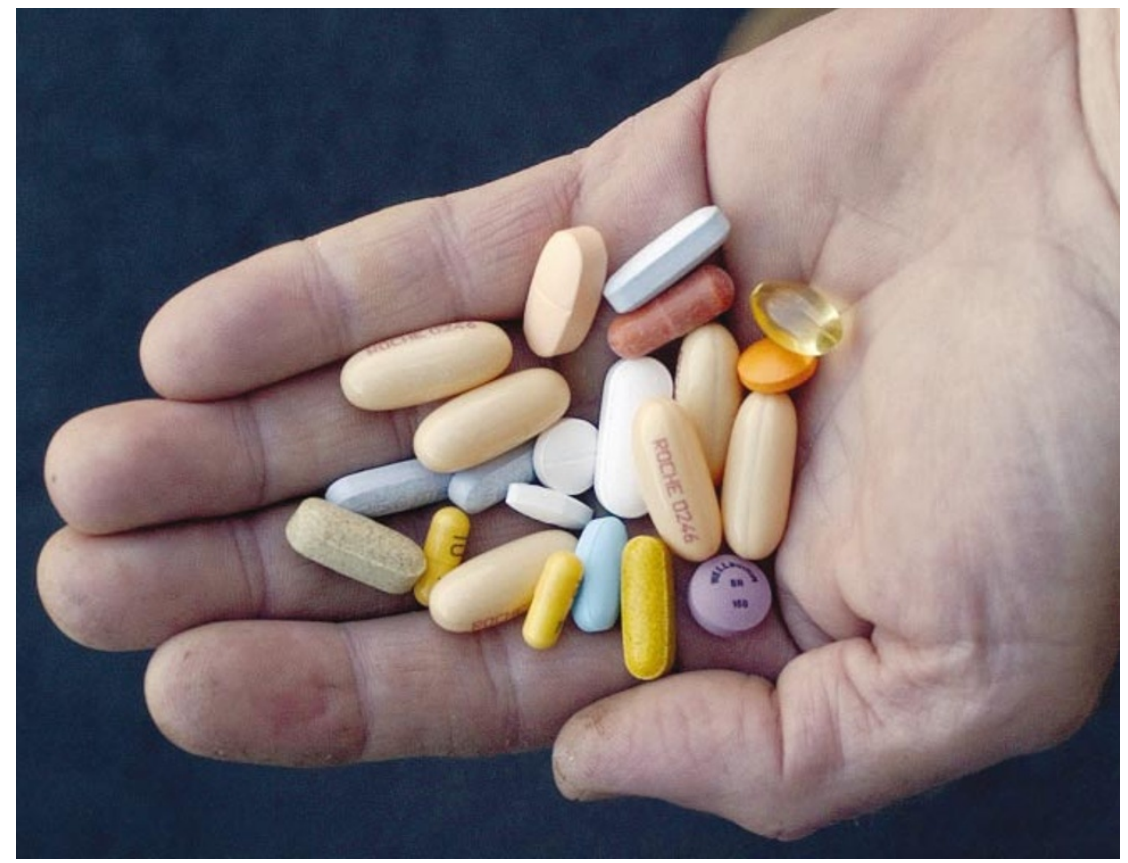

Hand of fate: HIV patients who don't stick to drug regimens seem not to foster resistant viral strains. data still show that patients who adhere better to their drug regimens live longer. Rather, researchers say, the findings indicate that patients in developing countries should be given access to the most effective drugs early in their treatment, because this maximizes their chances of controlling the virus. Patients who control the virus successfully do not develop drug resistance.

In patients who are taking drugs but are unable to control the virus, the virus is thought to develop mutations that allow it to survive in the presence of the drugs. Such mutants do not survive in patients whose drugs keep the virus in check.

"The clear message is that in the absence of complete viral suppression, the more medication you take, the more resistance you select," says Daniel Kuritzkes, director of AIDS research at Brigham and Women's Hospital in Boston, Massachusetts, and an author of an earlier study that suggested a link between less-effective therapies and resistance (D. R. Kuritzkes et al. J. Acquir. Immune Defic. Syndr. 23, 26-34;2000).

Researchers also say that data on patients in poor countries show that they are no less likely than those in rich countries to adhere to treatment. "There's no evidence to suggest that adherence is more of a challenge or resistance is more of a problem in the developing world, and there is no reason to delay the rollout of potent anti-retroviral therapies in resource-poor settings," Kuritzkes says.

\section{Water worlds make a splash as the best hope for alien life}

\section{Quirin Schiermeier, Munich}

Kevin Costner's 1995 film Waterworld might have flopped at the box office, but researchers think that real water worlds — Earth-sized planets predominantly covered by oceans - are more likely than land-covered planets to host life.

Simple assumptions about the likely distribution of planets in the Milky Way suggest that many water worlds exist in our Galaxy, but elude existing methods of detection. "There could be as many as one billion stellar systems with potentially habitable zones," says Siegfried Franck, a geophysicist at the Potsdam Institute for Climate Impact Research in Germany.

To try to pin down the locations of planets that might host life, Franck and Manfred Cuntz, an astrophyicist at the University of Texas in Arlington, used a mathematical model to locate the 'habitable zone' of $47 \mathrm{UMa}$, a Sun-like star some 45 light years away. The pair devised equations coupling stellar age and luminosity, distance from the star, and planetary climate, to determine the chance of habitable planets existing near 47 UMa. They also calculated geodynamic constraints on the biospheres of planets that could have formed there. (S. Franck et al. Int. J. Astrobiol. 2, 35-39; 2003).

Earth-like planets in stable orbits in habitable zones are the most likely places to harbour life. "Earth would have a slight chance of being habitable in the 47 UMa system," says Franck, "but a water world almost entirely covered by oceans would have a better chance."

The 47 UMa system intrigues experts because the star has roughly the same mass, age and spectrum as the Sun. Moreover, it hosts two giant gas planets, analogous to Jupiter and Saturn. It is thought that such large planets help to shelter Earth from bombardment by comets and asteroids.

"Studies like this help to publicize the notion of habitable zones," says Jim Kasting, an atmospheric scientist at Pennsylvania State University. But he warns that "models of early planetary evolution are not particularly well constrained" and may not provide a reliable pointer to where inhabitable planets can be found.

NASA plans to launch two space-based telescopes, perhaps by 2013, dedicated to the pursuit of Earth-like planets, and to the analysis of their atmospheric composition. "Then the whole thing will get really exciting," says Kasting. 